\title{
A Study on the Binding of Loperamide to Human Serum Albumin Using Combination of Computational and Experimental Methods
}

Komail Sadrjavadi ${ }^{1}$, Fatemeh Rahmati ${ }^{1}$, Fataneh Jafari ${ }^{1}$, Sajad Moradi ${ }^{2}$, Amin Nowroozi $^{1}$ and Mohsen Shahlaei ${ }^{3 *}$

${ }^{1}$ Pharmaceuticas Sciences Research Center, School of Pharmacy, Kermanshah University of Medical Sciences, Kermanshah, Iran

${ }^{2}$ Nano Drug Delivery Research Center, School of Pharmacy, Kermanshah University of Medical Sciences, Kermanshah, Iran

${ }^{3}$ Department of Medicinal Chemistry, Faculty of Pharmacy, Kermanshah University of Medical Sciences, Kermanshah, Iran

\begin{abstract}
In the present study, an attempt has been made to study the interaction of Loperamide, an drug used for treating symptoms or diarrhoea, with the most important transport protein, human serum albumin (HSA) employing UV-Vis, spectrofluorometric, FT-IR, Cyclic voltammetry, Electrochemical Impedance Spectroscopy and molecular modelling techniques. The results showed that binding of LOP to HSA caused strong fluorescence quenching of HSA through static quenching mechanism. The results of experimental and theoretical methods indicated that the binding of this drug to HSA induced slight conformational changes in HSA. Furthermore, the study of molecular modelling also showed that LOP could strongly bind to the site I (subdomain IIA) of HSA and hydrophobic interactions are the major forces in the stability of LOP-HSA complex.
\end{abstract}

Keywords: Loperamide; Protein binding; Fluorescence quenching; Human serum albumin

\section{Introduction}

Loperamide hydrochloride (Scheme 1) (LOP), has the IUPAC name 4-[4-(4-chlorophenyl)-4-hydroxypiperidin-1-yl]-N,N-dimethyl2,2-diphenylbutanamide hydrochloride $\left(\mathrm{C}_{29} \mathrm{H}_{33} \mathrm{ClN}_{2} \mathrm{O}_{2}\right)$ [1]. As it can be seen LOP is a piperidine derivative and opioid agonist with antidiarrheal activity for oral use. The mechanism of action of LOP is based on interaction of drug with mu receptors in the intestinal mucosa. This interaction leads to a decrease in gastrointestinal motility by decreasing the circular and longitudinal smooth muscle activity of the intestinal wall. This slows intestinal transit and allows for more water and electrolyte absorption from the intestines [2]. LOP is approved for the control of diarrhea symptoms and it is also available without a prescription (Figure 1).

In the literature, it is also reported that LOP using a number of different ways leads to decrease peristalsis and fluid secretion, resulting in longer gastrointestinal transit time and increased absorption of fluids and electrolytes from the gastrointestinal tract [3]. LOP has potential therapeutic usefulness as a peripherally selective topical or local opiate anti-hyperalgesic agent that lacks many of the side effects associated with opiate administration [4].

In humans, following oral administration of LOP about $50 \%$ of the administered LOP is absorbed in the gastrointestinal tract, absorbance occurring within $1 \mathrm{~h}$ in high bounding percent to plasma proteins (95\%) [5]

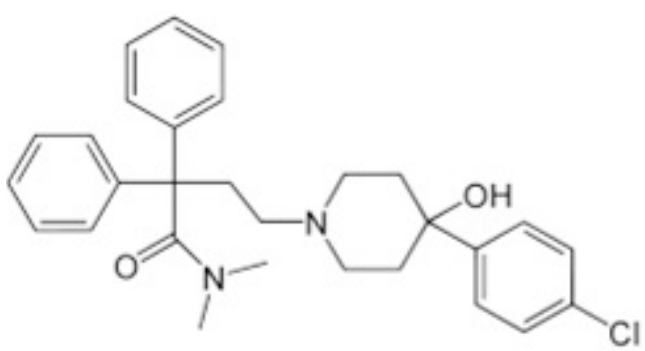

Figure 1: 2D chemical Structure of Loperamide (LOP).
HSA a single-chain, non-glycosylated protein with a molecular weight of $66,500 \mathrm{Da}$ containing 585 amino acids [6] is a major plasma polypeptide and is one of the most abundant carrier proteins which plays an important role in transport and disposition of endogenous and exogenous compounds present in blood [7]. It has been shown that the distribution, free concentration and the metabolism of various drugs can be significantly altered as a result of their binding to HSA [8].

Serum albumin is one of the most abundant carrier proteins which plays an important role in transport and disposition of endogenous and exogenous compounds present in blood.

As LOP is used to treat sudden diarrhoea widely, so it becomes necessary and important to carry out detailed investigation on the binding affinities and binding mechanism of LOP to the HSA in order to know the transport mechanism as well as to understand the effect of the drug on HSA structure.

The first 3D crystal structure of serum albumin protein with high resolution ( $2.8 \AA$ ) was resolved in 1998 by curry, et al. [9]. Subsequently, the structures of HSA forming complexes with other compounds have been popular and extensively studied by other groups, such as our research group [10-12]. The well-characterized structure and folding of the HSA will help comprehend the interaction of LOP with serum albumin which in turn will shed light to biological functioning of LOP. So, the present research of investigating the interaction of LOP with the most important transport protein in plasma, HSA, has been undertaken. To the best of our knowledge, no report has been published on LOP

*Corresponding author: Shahlaei M, Department of Medicinal Chemistry, Faculty of Pharmacy, Kermanshah University of Medical Sciences, Kermanshah, Iran, Tel +98-83-34276489; Fax: +98-831-34276493; E-mail: mohsenshahlaei@yahoo.com; mshahlaei@kums.ac.ir

Received: November 15, 2017; Accepted: December 06, 2017; Published December 15, 2017

Citation: Sadrjavadi K, Rahmati F, Jafari F, Moradi S, Nowroozi A, et al. (2017) A Study on the Binding of Loperamide to Human Serum Albumin Using Combination of Computational and Experimental Methods. Biochem Anal Biochem 6: 346. doi: 10.4172/2161-1009.1000346

Copyright: (c) 2017 Sadrjavadi K, et al. This is an open-access article distributed under the terms of the Creative Commons Attribution License, which permits unrestricted use, distribution, and reproduction in any medium, provided the original author and source are credited. 
interaction with serum albumin hinting at the transportation pathway of LOP through biological fluids, till date.

Here a combination of spectroscopic and electrochemical methods was applied to comprehensively investigate the interaction between the LOP and HSA. The possible conformational changes of the HSA upon binding of LOP have also been studied using the molecular docking and the molecular dynamics simulation methods.

This research, hopefully, will not only make available useful data for appropriately understanding the spectroscopic and electrochemical behaviour of the LOP, but also illustrate its binding affinity and binding mechanism at the atomistic level.

\section{Material and Methods}

\section{UV-Vis absorbance and circular dichroism spectral measure- ments}

Stock solution of Human serum albumin (fatty acid free), which was purchased from Sigma Chemical Co. and used without further purification, $\left(1 \times 10^{-3} \mathrm{M}\right.$, based on its molecular weight of 66,000$)$ was prepared in $0.01 \mathrm{M}$ phosphate buffer of $\mathrm{pH} 7.2$ and was kept in 277 K. Double distilled water was used throughout all experiments and measurements.

The UV spectra were then recorded at room temperature on an Agilent 8453 spectrophotometer equipped with a thermostatted bath and with a $1.0 \mathrm{~cm}$ quartz cuvette (Waldbornn, Germany). The absorbance measurements were recorded by keeping the serum albumin concentration constant $\left(3.0 \times 10^{-6} \mathrm{~mol} \mathrm{~L}^{-1}\right)$ while varying the LOP concentration (from $3.0 \times 10^{-6}-2.1 \times 10^{-5} \mathrm{~mol} \mathrm{~L}^{-1}$ ). The samples were scanned in the range $200-450 \mathrm{~nm}$, and the absorbance spectra were read every $1 \mathrm{~nm}$ in the absorption range.

Circular dichroism spectra were measured and recorded on a Jasco-810 automatic recording spectrophotometer, using a rectangular quartz cell of $2 \mathrm{~mm}$ cell length at room temperature. The experiments were carried out in the recording range of 200-260 $\mathrm{nm}$ with a scanning speed of $50 \mathrm{~nm} / \mathrm{min}$ and a response time of $4 \mathrm{~s}$. It must be noted that LOP itself did not affect the CD signal of HSA under these conditions. Titrations of HSA with LOP, for recording CD spectra were performed with a fixed concentration of HSA $\left(1.5 \times 10^{-6} \mathrm{M}\right)$ and the increasing concentrations of LOP $\left(1.5 \times 10^{-6} \mathrm{M}\right.$ to $\left.3 \times 10^{-6} \mathrm{M}\right)$.

\section{FTIR experiments}

FT-IR measurements were performed on Shimadzu FT-IR spectrometer (Japan) equipped with a Germanium attenuated total reflection (ATR) accessory and a DLaTGS detector a $\mathrm{KBr}$ beam splitter at room temperature. A 64 scan interferogram with $4 \mathrm{~cm}^{-1}$ resolution were used to collect the spectra. In the first step, the spectra of buffer and LOP solutions were collected under the same conditions as backgrounds. Then, the absorbance of the HSA in the absence and presence of the drug in the buffer solution was recorded. The LOPHSA complex solutions were prepared by mixing the proper amount of LOP and HSA up to final ligand protein molar ratios of 1:1 and 2:1. The background was corrected before scanning the samples and the buffer spectrum collected. Blank spectra were taken to subtract them from the LOP-HSA combined spectra.

\section{Steady-state fluorescence experiments}

Fluorescence spectra and intensities were recorded with LS-45 fluorescence spectrometer (Perkin-Elmer, U.K) using an excitation wavelength of $290 \mathrm{~nm}$ in a $1 \mathrm{~cm}$ quartz cell. Both excitation and emission slits were set at $10 \mathrm{~nm}$. The excitation wavelength was $280 \mathrm{~nm}$, and the emission wavelength was read at $320-420 \mathrm{~nm}$ with a maximum observed at $345 \mathrm{~nm}$.

The background fluorescence was subtracted from each emission spectrum. To determine the quenching mechanism as well as major binding forces, fluorescence measurements were performed at different temperature; 288, 298 and $308 \mathrm{~K}$. During the fluorescence titration experiments, $3.0 \mathrm{~mL}$ of solution containing the appropriate concentration of serum albumin was titrated manually by the successive addition of LOP with trace syringes. The fluorescence intensity was subsequently recorded.

\section{Determination of binding constant and number of binding sites}

The fluorescence emission intensities for serum albumin, measured at $345 \mathrm{~nm}$ as a function of increasing concentrations of the LOP, were used for the construction of the binding profiles and the fluorescence quenching mechanism.

The decrease in fluorescence intensity at $\lambda$ max at two temperatures $(288,298$ and $308 \mathrm{~K})$ was studied using the well-known Stern-Volmer equation:

$$
F_{0} / F=1+K_{S V}[Q]=1+k_{q} \tau_{0}[Q]
$$

where $\mathrm{F} 0$ and $\mathrm{F}$ are the fluorescence intensities of serum albumin in the absence and presence of the LOP as quencher, respectively, [Q] is the concentration of the LOP and KSV, kq and $\tau 0$ are the Stern-Volmer quenching constant, quenching rate constant and fluorescence lifetime (for HSA, the lifetime of the fluorophore is approximately $1 \times 10^{-8} \mathrm{~s}$ ), in the absence of LOP, respectively. As can be concluded from above equation, KSV can be determined by linear regression of a plot of F0/F vs. [Q].

\section{Dynamic light scattering measurements}

Dynamic light scattering (DLS) approach was used to measure the hydrodynamic radius $(\mathrm{Rh})$ of the prepared samples (Malvern zetasizerNano ZS) at $25^{\circ} \mathrm{C}$ and $633 \mathrm{~nm}$. Mean hydrodynamic radius values were estimated using following equation (Stokes-Einstein equation):

$$
R h=\frac{k T}{6 \pi \eta D}
$$

In the above equation, the $\mathrm{Rh}$ is the hydrodynamic radius, $\mathrm{K}$ represent Boltzmann constant, $\mathrm{T}$ is temperature based on Kelvin, $\eta$ denotes the viscosity of water, and $\mathrm{D}$ is diffusion coefficient.

\section{Molecular docking}

The structure of HSA was downloaded from Brookhaven Protein Data Bank (see http://www.rcsb.org/pdb) with PDB ID (1AO6). In order to calculate the optimized and stable conformation of LOP, the 2D structure of LOP was drawn by Chem Office 2015 and then was transferred to Hyperchem. The 3D structure was energetically minimized by a semi empirical method (PM3), implemented in HyperChem. In the next step, the geometry with the lowest total energy was chosen for docking procedure. Docking simulation was carried out using AutoDock 4.2 program package [13]. Firstly, Rotatable bonds in the Clo were assigned with AutoDock Tools [14]. Then, AutoDock Tools was employed for the preparation of PDBQT files of LOP and HSA. It must be noted that at first, and before adding torsional parameters and charge to structures, water molecules were removed from the pdb file of protein. In the following, the Gastieger charges 
and missing hydrogen atoms were added to the protein structure using MGL Tools. Grid maps were calculated with $0.375 \AA$ spacing by the Auto Grid program for the whole protein target. The Grid Maps were generated for various ligand's atom types, such as aliphatic carbons, aromatic carbons, hydrogen bonding oxygens, and so on. Also, the partial charges of atoms were calculated using Gasteiger-Marsili procedure [15]. After determining Kollman united atom charges [16].

As a final step, docking of LOP to the HSA has been performed applying AutoDock. In this study docking experiments were performed using the AutoDock empirical free energy scoring function and the Larckian genetic algorithm with local search [17]. The number of independent docking runs carried out for each docking procedure was assigned to 250 . For each independent run 25,000,000 energy was evaluated. Other docking parameters were set to the default values. The center of the grid was set at the Ca atom of Trp214 with respect to fluorescence results. Binding pocket was enclosed in a box with the number of grid points in $\mathrm{x} \times \mathrm{y} \times \mathrm{z}$ directions, $60 \times 60 \times 60$ and a grid spacing of $0.375 \AA$, to allow the ligand to rotate freely. Finally, docking results were investigated using VMD 1.8.6 [18].

\section{Molecular dynamics simulation}

To verify the main binding mode of LOP and important interactions and to study the overall effect of ligand to the serum albumin, an MD simulation was carried out.

GROMOS96 force-field was applied to MD simulation using through the GROMACS software (Ver. 5, www.gromacs.org) [19]. The structure of complex of LOP and HSA obtained from the docking procedure was used as starting point for the MD simulation. HSA and lop were soaked in a cubic box of SPC (Single Point Charge) water molecules [20] and simulated using periodic boundary conditions. During the $50 \mathrm{~ns}$ MD simulation was carried in the isobaric-isothermal ensemble (NPT: $300 \mathrm{~K}$ and 1 bar). Using the productive MD, pressure was kept constant at 1 bar by altering the box dimensions and the time-constant for pressure coupling was set to 1 Ps [10]. The topology parameters and topology file of protein were generated by using the Gromacs program (pdb2 gm x command). The force field parameters for LOP were calculated using Automated Topology Builder (ATB) and Repository [21].

\section{Electrochemical apparatus and conditions}

Electrochemical experiments were carried out using a $\mu$-Autolab TYPE III (Eco Chemie B.V., Ultrecht, The Netherlands) potentiostat/ galvanostate and equipped with NOVA software (Version 1.11).This instrument also was equipped with a conventional three electrode cell, an $\mathrm{Ag} / \mathrm{AgCl}$ in $\mathrm{KCl}\left(3 \mathrm{~mol} \mathrm{~L}^{-1}\right)$ as reference electrode, a Pt electrode as counter electrode, a carbon paste electrode (CPE) modified by $\mathrm{TiO}_{2}$ working electrode ( $1.2 \mathrm{~mm}$ diameter).and a personal computer for data storage and processing. All potentials were referred to an $\mathrm{Ag} / \mathrm{AgCl} /$ $\mathrm{KCl}\left(3 \mathrm{~mol} \mathrm{~L}^{-1}\right)$ electrode. All electrochemical measurements were performed at room temperature.

All reagents and chemicals were of analytical grade and used as received. The used carbon paste electrode modified with $\mathrm{TIO}_{2}$ nanoparticles was prepared by mixing $\mathrm{TIO}_{2}$ and noparticles and Nujol in an agate mortar with pestle. After preparation of mixture, it was packed into a glass tube $(\varphi=1.2 \mathrm{~mm})$, and a copper wire was embedded in the paste for electrical connection.

At the electrochemical experiments, at the first the electrolyte contains $5 \times 10^{-3} \mathrm{~mol} \mathrm{~L}^{-1} \mathrm{~K}_{3} \mathrm{Fe}(\mathrm{CN})_{6} / \mathrm{K}_{4} \mathrm{Fe}(\mathrm{CN})_{6}$ and $10 \times 10^{-1} \mathrm{~mol} \mathrm{~L}^{-1}$ $\mathrm{KCl}$ at $\mathrm{pH}=7.4$ was used as a Redox probe. In the following step, 10
$\mathrm{mL}$ electrolyte was added into the electrochemical cell with a magnetic stirring device. The measurements were carried out after suitable amount of protein solution was added to electrochemical cell to reach to $1 \times 10^{-5} \mathrm{M}$. The electrodes were immersed for a couple of minutes before obtaining the cyclic voltammograms and other information. Then different volumes of drug solution were added continuously to the electrochemical cell and stirred for two minutes before recording results.

The Electrochemical impedance spectroscopy (EIS) measurements were carried out in order to investigate the barrier properties of LOPHSA complex. Also, The EIS measurements are often employed to determine the amount of "Redox" probe that associated with changes in the capacitance and/or electron transfer resistance at the working electrode surface. Thus, impedance behavior of LOP-HSA complex deposits was investigated by Nyquist plot in the frequency range from $100 \mathrm{mHz}$ to $100 \mathrm{kHz}$ and in a potential of $+0.25 \mathrm{~V} \mathrm{Ag} / \mathrm{AgCl}$. In the obtained results, the particular semicircle diameter relates to the charge transfer resistance, Rct, the values of which are calculated using the fitting program NOVA software.

\section{Results and Discussion}

\section{UV-vis absorbance experiments}

To study the structural change of HSA by binding of LOP, UVVis experiments, as a simple but effective technique for detecting complex formation, were used. In all UV-Vis experiment, constant concentration of HSA and different amounts of LOP were used. Concentration of LOP was increased progressively to the $3.0 \times 10-6 \mathrm{M}$ serum albumin solution to achieve ligand/protein ratios from 1.0 to 7 $((\mathrm{ri}=[\mathrm{LOP}] /[\mathrm{HSA}])=1.0,2.0,3.0,4.0,5.0,6.0,7.0))$ Figure 2 displays the UV-Vis spectra of HSA and different amounts of LOP. Because of the possible overlapping between the LOP absorbance spectrum and HSA absorbance bands, the effect of LOP on the UV-Vis curves was removed by employing corresponding concentration of ligand as reference solution during the recording spectra. As can be seen in the figure, serum albumin has a distinguished absorption peak at $\sim 280 \mathrm{~nm}$. The comparison of the absorbance spectra, before and after binding of LOP, demonstrates that formation of complex between HAS and LOP causes a slight hypochromic effect. This phenomena implies the presence of a specific interaction between the LOP and HSA.

\section{Circular dichroism}

CD spectra, which are the difference in the absorption of lefthanded circularly polarised light and right-handed circularly polarised

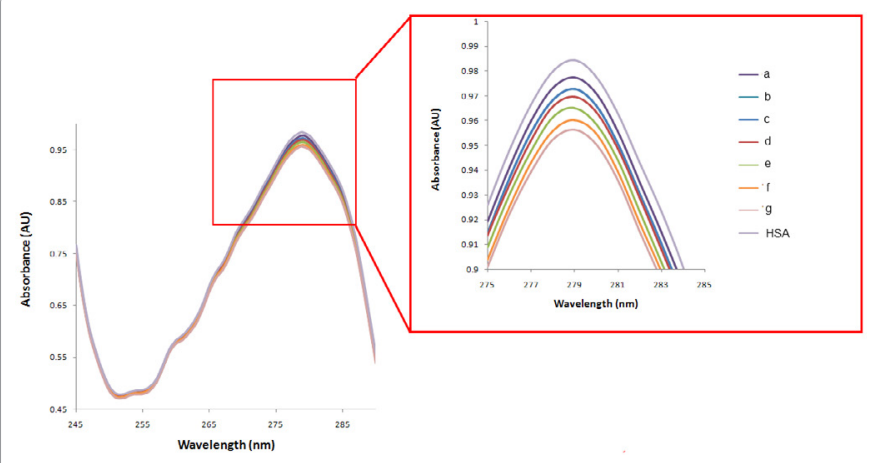

Figure 2: UV absorbance of HSA $\left(3.0 \times 10^{-6} \mathrm{M}\right)$ with different concentration of LOP (a) $3 \times 10^{-6} \mathrm{M}$ (b) $6 \times 10^{-6} \mathrm{M}$ (c) $9 \times 10^{-6} \mathrm{M}$ (d) $1.2 \times 10^{-6} \mathrm{M}$ (e) $1.5 \times 10^{-5} \mathrm{M}$ (f) $1.8 \times 10^{-5} \mathrm{M}(\mathrm{g}) 2.1 \times 10^{-5} \mathrm{Mat} \mathrm{pH}=7.4$. 
light, of HSA show two distinct negative bands in the ultraviolet region at 209 and $221 \mathrm{~nm}$ as shown in Figure 3.

This is characteristic of the helical structure of a protein. As it can be found by examining the results by this method, it was found that the binding of LOP to serum albumin causes a slight geometrical change of the protein, leading to a loss in its helical content.

Circular dichroism (CD) is also a helpful approach for studying geometrical changes of proteins upon interaction with small ligands like drugs. So, the conformation of the complex formed between HSA and LOP has been further studied using CD method. HSA has a high percentage of $\alpha$-helical structure which indicates characteristic strong double minimum signals at 222 and $208 \mathrm{~nm}$ [22] (Figure 3). The intensities of the two double minimum signals reflect the amount of helicity of serum albumin. Upon addition of the LOP to serum albumin, dichroic spectra of HSA in the studied region $(200-250 \mathrm{~nm})$ show a distinct change in the presence of LOP which imply that the extent of a-helicity of the protein decreased and hence, the intensity of the double minimum was reduced. This is an implication of change in helicity when the LOP is completely connected to protein. The CD spectra of HSA in the presence and absence of the LOP were observed to be similar in shape, which meant that the structure of serum albumin was also predominantly $a$-helix [23]. Therefore, it can conclude that the binding of LOP to the binding site of HSA induced small changes in secondary-structure of protein. These results are in agreement with the UV-Vis absorption spectra results.

\section{Analysis of fluorescence quenching}

Fluorescence quenching can be explained as a process of the decrease of the quantum yield of fluorophore (here serum albumin) induced by a variety of molecular interactions with a quencher molecule (LOP in this study). Basically, the fluorescence of proteins resulted from tryptophan, tyrosine and phenylalanine residues. Specially, the inherent fluorescence of HSA is almost always because of tryptophan residue. It must be noted that phenylalanine has a very low quantum yield and the fluorescence of tyrosine is nearly totally quenched if it is ionized or near to an amino group, a carboxyl group or a tryptophan [24]. So, the natural intensity attributes to their fluorescent tryptophan residues. It is reported that most of fluorescence intensity of proteins are occurred around $348 \mathrm{~nm}$ [25]. To be exact, the change of natural fluorescence intensity of serum albumin was caused by interaction of small ligands such as drugs to tryptophan residue of HSA. When different concentrations of LOP solution were titrated with a fixed amount of serum albumin, a significant decrease in the fluorescence intensity of protein was observed as it is shown in Figure 4A.

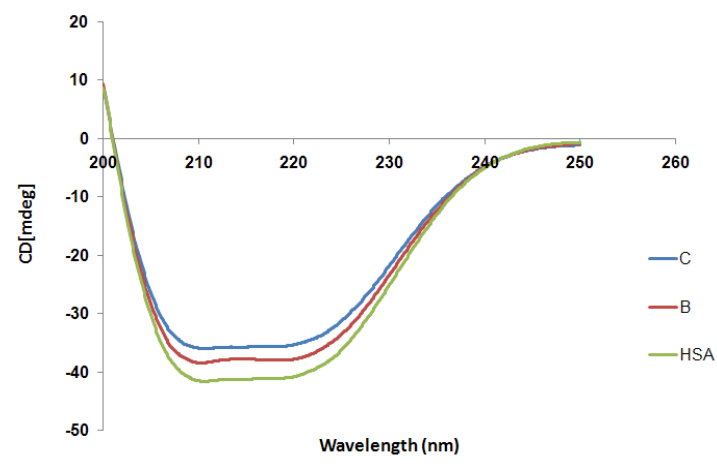

Figure 3: CD spectra of the LOP-HSA complex at different drug to HSA ratios at $\mathrm{pH} 7.4$ and $298 \mathrm{~K}$. Drug to HSA $\left(1.5 \times 10^{-6} \mathrm{~mol} / \mathrm{L}\right)$ ratios: $(B) 1: 1$ (C) $2: 1$.
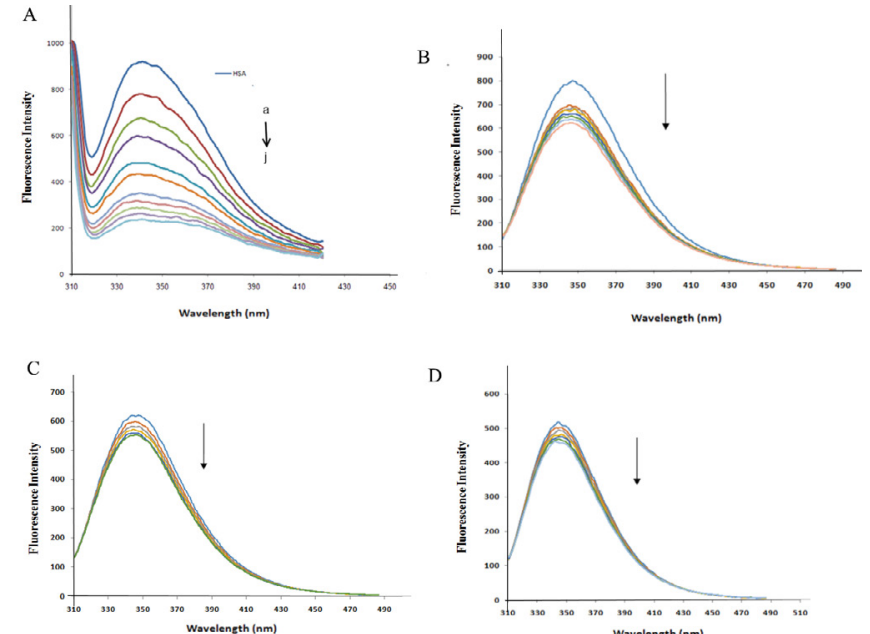

Figure 4: (A) Fluorescence spectra of HSA in the absence and the presence of LOP. The fluorescence quenching spectra of HSA by different concentrations of LOP in Tris-HCl buffer, $\mathrm{pH} 7.4$, at different temperatures: (B) $308 \mathrm{~K}$ (C) $298 \mathrm{~K}$ (D) $288 \mathrm{~K}$. [HSA], $1.0 \times 10^{-6} \mathrm{M}$; the concentration of LOP corresponding is gradually increased. Arrow shows the intensity changes upon increasing concentration of the LOP.

Fluorescent quenching phenomena involve a variety of molecular interactions such as excited state reactions, molecular rearrangements, energy transfer, the formation of ground-state complex, collision, etc. [26]. Therefore, fluorescence quenching measurement were carried out to study the interaction between LOP and serum albumin. When the serum albumin is excited at $285 \mathrm{~nm}$, both tryptophan and tyrosine amino acid residues existed in structure of HSA provide fluorescence emissions in which tryptophan has strong fluorescence emissions in comparison to tyrosine which does not absorb electromagnetic radiation this region [27]. The fluorescence spectra of serum albumin in the absence and presence of LOP as a quencher in Tris- $\mathrm{HCl}$ buffer ( $\mathrm{pH}$ 7.4) were traced (Figure 4A). As it can be seen, serum albumin shows a strong fluorescence emission with a peak at about $345 \mathrm{~nm}$ due to the emission from the single tryptophan residue (Trp-214). However, intrinsic fluorescence intensity of serum albumin diminished regularly and additionally. Also, a slight shift of the emission maximum from 346 to $343 \mathrm{~nm}$ is detected (Figure 4A). This evidence denotes the change in solvent accessibility in the serum albumin resulted from interaction of LOP to a binding pocket in the vicinity of aromatic fluorophores particular Trp-214. The strong quenching of the serum albumin fluorescence proved the existence of interaction between LOP and serum albumin which changes the local environment around Trp214 residue [10]. With the intention of determine the fluorescence quenching mechanism, the fluorescence quenching data at different temperatures $(288,298$ and $308 \mathrm{~K}$ ) (Figure 4B-4D) were analysed using the classical Stern-Volmer equation (as discussed above).

To further describe the fluorescence quenching mechanism, SternVolmer equation was used. The Stern-Volmer plot of the titrations of serum albumin by LOP is represented in Figure 5 and the calculated KSV and kq values are summarized in Table 1 . At three studied temperatures $(288,298$ and $308 \mathrm{~K})$ Stern-Volmer plots have the same trends. At all studied concentrations of LOP, linear plots were produced.

The results revealed that the Stern-Volmer quenching constant $\mathrm{K}_{\mathrm{sV}}$ is inversely correlated with temperature and $\mathrm{K}_{\mathrm{g}}$ is larger than the limiting diffusion constant $\mathrm{K}_{\mathrm{dif}}$ of the biomolecule $\left(\mathrm{K}_{\mathrm{dif}}=2.01010 \mathrm{M}^{-1} \mathrm{~s}\right.$ 
$\left.{ }^{-1}\right)$ [26] showing that fluorescence quenching was caused by a specific interaction between serum albumin and LOP, consistent with the static quenching mechanism.

In the linear range of Stern-Volmer curve, the number of binding sites was obtained according to eqn. (1). From the slope of the regression curve based on Stern-Volmer equation (eqn. (1)), the association constant $\left(\mathrm{K}_{\mathrm{a}}\right)$ and the number of binding site $(\mathrm{n})$ can be obtained from the regression by eqn. (1). The values of $\mathrm{K}_{\mathrm{a}}$ and $\mathrm{n}$ are 7.7 $\times 10^{5} \mathrm{M}^{-1}$ and 1.8 at $298 \mathrm{~K}$, respectively.

In the case of static quenching, same as this case, the quenching must be further studied according to the following equation that called modified Stern-Volmer equation [28]:

$$
\frac{\mathrm{F}_{0}}{\left(\mathrm{~F}_{0}-\mathrm{F}\right)}=\frac{1}{\mathrm{f}_{\mathrm{a}} k_{a}} \frac{1}{[\mathrm{Q}]}+\frac{1}{\mathrm{f}_{\mathrm{a}}}
$$

where, $\mathrm{F}_{0}-\mathrm{F}$ is the difference in fluorescence intensity in the presence

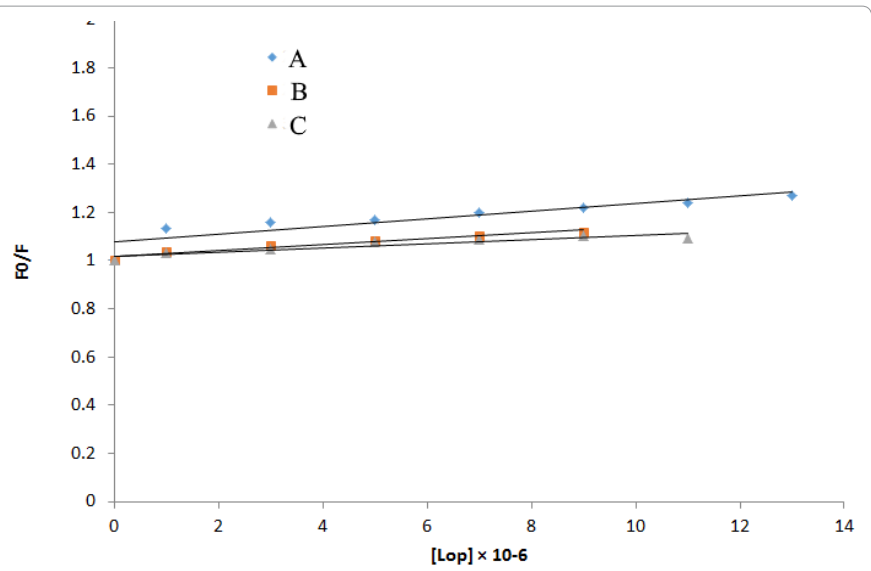

Figure 5: The Stern-Volmer plots at different temperatures (A) $288 \mathrm{~K}$ (B) $298 \mathrm{~K}$

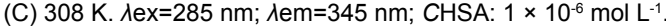

\begin{tabular}{|c|c|c|c|c|c|}
\hline $\begin{array}{c}\mathbf{T} \\
(\mathbf{K})\end{array}$ & $\begin{array}{c}\mathrm{K}_{\mathrm{sv}} \times 10^{4}(\mathrm{~L} \\
\left.\mathrm{mol}^{-1}\right)\end{array}$ & $\begin{array}{c}\mathrm{K}_{\mathrm{q}} \times 10^{12}(\mathrm{~L} \\
\left.\mathrm{mol}^{-1}\right)\end{array}$ & $\mathrm{K}_{\mathrm{b}} \times \underset{\left.\mathrm{L}^{-1}\right)}{10^{5}}(\mathrm{~mol}$ & $\mathrm{n}$ & $\begin{array}{c}\mathrm{K}_{\mathrm{a}} \times 10^{-4}(\mathrm{~L} \\
\left.\mathrm{mol}^{-1}\right)\end{array}$ \\
\hline 288 & 1.5 & 1.5 & 4.91 & 0.691 & 1.48 \\
\hline 298 & 1.2 & 1.2 & 0.778 & 1.891 & 0.365 \\
\hline 308 & 0.8 & 0.8 & 0.927 & 1.967 & 0.366 \\
\hline
\end{tabular}

Table 1: The Stern-Volmer quenching constant $(K$ ). The quenching constants $(K q)$, binding constants $\left(K_{b}\right)$, number of binding sites $(n)$ and the effective quenching constant for the LOP-HSA system at different temperatures.

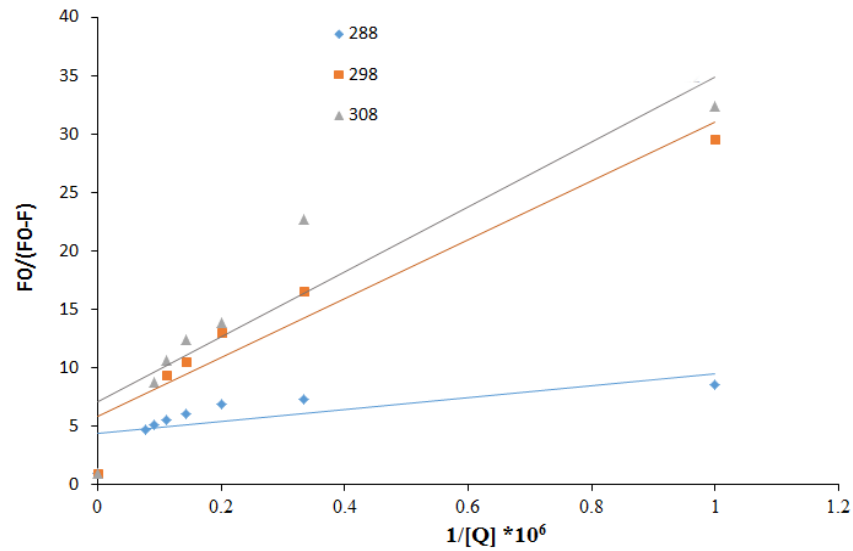

Figure 6: The modified Stern-Volmer plots for the LOP-HSA complexes at $(\star)$ $288 \mathrm{~K}(\mathrm{\Xi}) 298 \mathrm{~K}$, and $\left({ }^{\wedge}\right) 308 \mathrm{~K},[\mathrm{HSA}]=1 \mu \mathrm{M}, \lambda=295 \mathrm{~nm}$ at $\mathrm{pH} 7.4$.

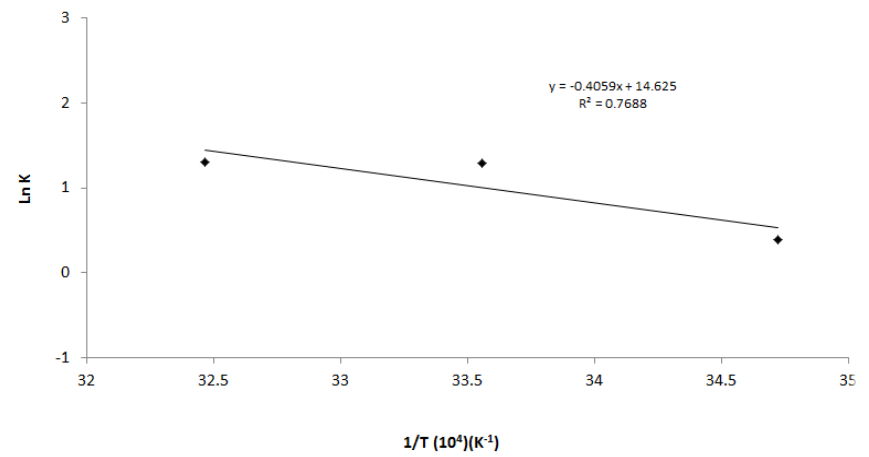

Figure 7: van't Hoff plot, $\mathrm{pH} 7.40, c(\mathrm{HSA})=1.0 \times 10^{-6} \mathrm{M}$

\begin{tabular}{|c|c|c|c|}
\hline $\mathbf{T}(\mathbf{K})$ & $\boldsymbol{\Delta} \mathbf{H}\left(\mathbf{J ~ m o l}^{-1}\right)$ & $\boldsymbol{\Delta S}\left(\mathbf{J ~ m o l}^{-1} \mathbf{~ K}^{-1}\right)$ & $\boldsymbol{\Delta} \mathbf{G}\left(\mathbf{J ~ m o l}^{-1}\right)$ \\
\hline 288 & 0.34 & 0.0014 & -0.07 \\
\hline 298 & & & -0.08 \\
\hline 308 & & & -0.10 \\
\hline
\end{tabular}

Table 2: The thermodynamic parameters of the system of LOP-HSA.

$(F)$ of the LOP (In the present case, as quencher) at concentration $[Q]$ and in the absence of LOP $\left(F_{0}\right)$. The parameter ka is the effective quenching constant for the accessible fluorophores, which is analogous to associative binding constants for the quencher (LOP)-acceptor (HSA) system, and fa is the fraction of accessible fluorescence. The dependence of $\mathrm{F}_{0} / \Delta \mathrm{F}$ on the reciprocal value of the LOP concentration (as a quencher) $1 /[\mathrm{Q}]$ is the linear with slope equal to the value of $1 / \mathrm{f}_{\mathrm{a}} \mathrm{K}$. The value of $1 / f_{a}$ is fixed on the ordinate. The constant $K_{a}$ is the quotient of an ordinate $1 / f_{a}$ and slope $1 / f_{a} K_{a}$. Figure 6 shows the modified SternVolmer plot at different temperatures.

Analysis of the quenching data on the modified Stern-Volmer equation reveals the associative binding constant $\left(\mathrm{K}_{\mathrm{a}}\right)$ at the different experimental temperatures (Table 1)

\section{Binding mode}

The interacting forces between a small molecule and a biomacromolecule mainly include four main types of non-covalent interaction. These weak interactions are including hydrogen bonds, van der Waals forces, electrostatic and hydrophobic interactions [29].

The thermodynamic parameters of binding reaction between LOP and HAS such as enthalpy change $(\Delta \mathrm{H})$, entropy change $(\Delta \mathrm{S})$ and free energy change $(\Delta G)$ are the main evidences which can be used to determine the binding mode. Therefore, and for determining above parameters, the temperature-dependence of the binding constant was studied. The temperatures which are studied were 288,298 , and $308 \mathrm{~K}$. By plotting the binding constants $\left(\mathrm{K}_{\mathrm{b}}\right.$ values in Table 1$)$ according to the van't Hoff equation (eqn. (5)) the thermodynamic parameters can be calculated from a linear van't Hoff plot via Figure 7 and tabulated in Table 2.

$$
\ln K=\frac{-\Delta H}{R T}+\frac{\Delta S}{R}
$$

Where $\mathrm{K}$ is analogous to the effective quenching constants at the corresponding temperature and $\mathrm{R}$ is the gas constant.

From the thermodynamic standpoint, $\Delta \mathrm{H}>0$ and $\Delta \mathrm{S}>0$ imply a hydrophobic interaction; $\Delta \mathrm{H}<0$ and $\Delta \mathrm{S}<0$ reflect the van der Waals force or hydrogen bond formation; and $\mathrm{DH} \approx 0$ and $\Delta S>0$ suggest an electrostatic force. The thermodynamic temperatures chosen for measurements were 288,298 and $308 \mathrm{~K}$, so that HSA does not undergo 
any structural degradation. The thermodynamic parameters can be calculated from the Van't Hoff equation:

$$
\Delta G=\Delta H-T \Delta S=-R T \ln K
$$

The interacting forces between small molecules such as drugs and HSA show weak interactions such as electrostatic forces, hydrophobic interaction, hydrogen-bond formation, and van der Waals forces and stereo-hindrance effect [29]. In a typical binding procedure, the thermodynamic variables changes, such as enthalpy change $(\Delta \mathrm{H})$, entropy change $(\Delta S)$ and free energy change $(\Delta G)$ are the main factors for describing the binding modes and to determine the interactive forces. Based on the signs of these changes, a researcher can determines important interactions involved in binding process.

According to the obtained values for $\Delta \mathrm{H}$ and $\Delta \mathrm{S}$, the nature of the binding between LOP and protein can be elucidated [30]. If $\Delta \mathrm{H}>0$ and $\Delta S>0$, the main force of interaction is hydrophobic, if $\Delta \mathrm{H}<0$ and $\Delta S>0$, electrostatic attraction is the predominant force, and finally, if $\Delta \mathrm{H}<0$ and $\Delta \mathrm{S}<0$, both van der Waals forces and hydrogen bonding are established. The thermodynamic parameters for the interaction of LOP with serum albumin are also reported in Table 2. The negative sign of $\Delta \mathrm{G}$ means that the binding between LOP and serum albumin is spontaneous. Furthermore, as reported in Table 2, the formation of LOP-HSA coordination compound is an endothermic reaction accompanied by positive $\Delta S$ value which shows that hydrophobic forces play a major role in the binding between LOP and HSA.

\section{Stoichiometry of binding}

The stoichiometry of interaction between LOP and HSA was determined by the method of continuous variation or Job plot analysis. This method is a very beneficial approach for the characterization of complex resulted from an interaction of two species such as ligand and protein. The interpretation of results are very clear and straightforward, if, as in this case, the drug molecule has no UV/Vis abortion in in the range of wavelength scanned for HSA. Hence, the relative UV/Vis absorbance recorded is directly proportional to the HSA concentration as present in the complex. As it is obvious from the Figure 8, the obtained results showed that the cross point between the two straight lines occurs at a molar fraction (Xdye) around 0.5 , which suggests that the stoichiometric ratio of LOP: HSA at $25^{\circ} \mathrm{C}$ and $\mathrm{pH} 7.40$ is $1: 1$.

\section{Conformational changes studied by synchronous fluores- cence spectroscopy (SFS)}

It was established that binding of LOP to serum albumin results in the fluorescence quenching of protein, but it is still unclear that does this binding affects the conformation and/or the molecular

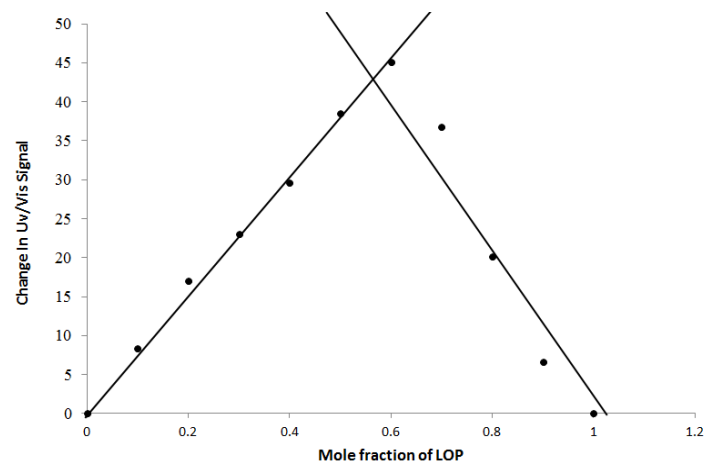

Figure 8: Job's plot based on the method of continuous variation. Showing stoichiometry of the LOP: HSA interaction.
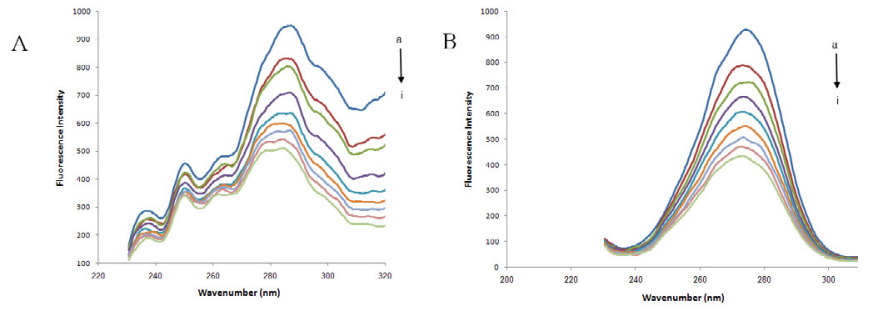

Figure 9: Synchronous fluorescence spectra of serum albumin with $\Delta \lambda=15$ $\mathrm{nm}(\mathrm{A}) \Delta \lambda=60 \mathrm{~nm}$ (B) and in the absence and presence of increasing amount of LOP. The ratio of CLOP/CHSA were $0,4,8,12,16,20,24,28$ and 32 ; $\mathrm{CHSA}=3.0 \times 10^{-7} \mathrm{M} ; \mathrm{pH}=7.4 ; T=298 \mathrm{~K}$

environment in a vicinity of serum albumin. With the purpose of further study the binding of LOP to serum albumin, the technique of Synchronous fluorescence spectroscopy (SFS) was applied. SFS, which involves the simultaneous scanning of excitation and the emission monochromators of a spectrofluorimeter as maintaining a fixed wavelength difference $(\Delta \lambda)$ between them, has been employed to investigate the microenvironment of amino acid residues of proteins [31]. SFS is a very efficient method to study the microenvironments around the fluorophore functional groups in proteins and other macromolecules. As discussed above, fluorescence of serum albumin arises from the tyrosine, tryptophan and phenylalanine residues. In the synchronous fluorescence spectra, the sensitivity associated with fluorescence approach is preserved as several advantages are also available: spectral simplification, spectral bandwidth reduction and avoidance of different perturbing effects [32]. According to Miller [33], the fluorescence of serum albumin with $\Delta \lambda\left(\Delta \lambda=\lambda_{\text {emission }}-\lambda_{\text {exitation }}\right)$ of 60 and $15 \mathrm{~nm}$ are main features of tryptophan and tyrosine residues, respectively. The effect of LOP on synchronous fluorescence spectra was shown in Figure 9. As it can be seen, the addition of the LOP causes the strong fluorescence quenching of tryptophan and tyrosine with the maximum emission wavelength at $274.5 \mathrm{~nm}$ and 287, respectively.

As it can be seen in Figure 8, the spectra evidence a shift from 274.5 $\mathrm{nm}$ to $273.5 \mathrm{~nm}$ for $\Delta \lambda=60$ and $287 \mathrm{~nm}$ to $285 \mathrm{~nm}$ for $\Delta \lambda=15$ showing that there are slight changes in conformation near tryptophan and tyrosine. These observations propose that LOP has slight effect on the microenvironment of the Tyr 263 residue in HSA that is comparable with that on the microenvironment of the Trp 214 residue. The results indicate that Tyr 263 in subdomain IIA is involved in binding. In addition, the small blue shifts in the spectra express a slight change in conformation of serum albumin upon binding with LOP.

\section{Excitation-emission matrix spectroscopy}

Excitation-emission matrix spectroscopy (EEMS) or 3D fluorescence spectroscopy is a new technique that can be used to present information regarding the conformational and structural change of protein bound to a ligand through a comprehensive display of the fluorescence characteristics of the sample under study both as a function of excitation and emission wavelengths simultaneously $[20,29,33]$. The excellent advantage of $3 \mathrm{D}$ fluorescence spectra is that information concerning the fluorescence characteristics can be entirely obtained by simultaneously changing the excitation and emission wavelengths. So, this method has also been used in the present study to understand the conformational changes of protein in presence of LOP depicts the 3D fluorescence spectra of serum albumin in absence and presence of LOP. Figure 10A and 10C show the threedimensional fluorescence spectra and corresponding contour plots of free HSA and LOP-HSA complex, respectively. The 3D fluorescence 
spectra of serum albumin (Figure 10A) shows two detectable peaks denoted as peak I and peak II in the figure. Peak I principally showed the fluorescence behaviour of poly peptide backbone structure, and signified the changes in the secondary structure of HSA [34]. Peak II presented the intrinsic fluorescence of Trp and Tyr residues. A direct comparison of Figure 10A and 10C distinctly reveals that there occurs a small reduction in the peak I and peak II. The results implied in Figure 10 reveal that in LOP-HSA system the fluorescent intensity of peak II decreased, which showed that the polarity of both residues (Trp and Tyr) was reduced and that more residues of HSA were buried in the hydrophobic pocket. A lower polarity intended that the binding position between HSA and LOP was located within this hydrophobic pocket, and that the addition of LOP changed the polarity of this hydrophobic microenvironment and therefore also the conformation of serum albumin. When comparing the peak I for studied systems, it can be seen that the $\lambda$ ex, $\lambda$ em and intensity of peak I change in the presence of LOP, which implies that the protein backbone structure of HSA has been changed in the presence of ligand. The strength of peak I in the LOP-HSA complex was lower than in the free HSA. It indicates that the secondary structure of HSA has been slightly changed during the formation of the complex.

Thus, the present findings from EEMS confirm the conformational changes of serum albumin in presence of LOP, which also corroborates with other our findings from synchronous fluorescence and molecular dynamics simulation results.

\section{FT-IR analysis}

Additional evidence regarding the LOP-HSA complications comes from infrared spectroscopy results. The secondary structure of serum albumin is corresponded to amide I band ranging from 1600 to $1700 \mathrm{~cm}^{-1}$ (mainly C O stretch) and amide II band about $1548 \mathrm{~cm}^{-1}$ (C-N stretch coupled with $\mathrm{N}-\mathrm{H}$ bending mode) [35]. Figure 11 (blue line) showed the FT-IR spectrum of free serum albumin in Tris- $\mathrm{HCl}$ buffer, and the spectra of LOP-HSA at 1:1 ligand: HSA ratio were also displayed in Figure 11. The FT-IR spectra of serum albumin (Figure 11) imply the effect on the secondary structure of protein due to complex formation of LOP and HSA. The amide I of free serum albumin has shifted from $1643 \mathrm{~cm}^{-1}$ to $1651 \mathrm{~cm}^{-1}$ at $1: 1$ ligand: HSA ratio. The peak near $1543 \mathrm{~cm}^{-1}$ shifted to $1547 \mathrm{~cm}^{-1}$ indicating the effect on binding of
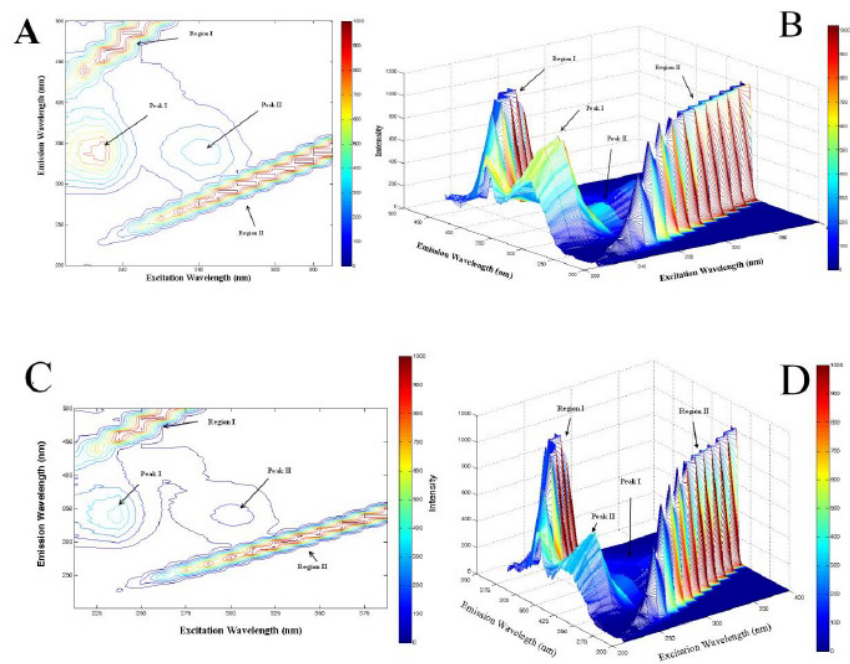

Figure 10: Three-dimensional fluorescence spectra and corresponding contour plots of $(A)$ and $(B)$ free HSA; (C and D) LOP-HSA complex $(\mathrm{pH}=7.4, T=298 \mathrm{~K})$

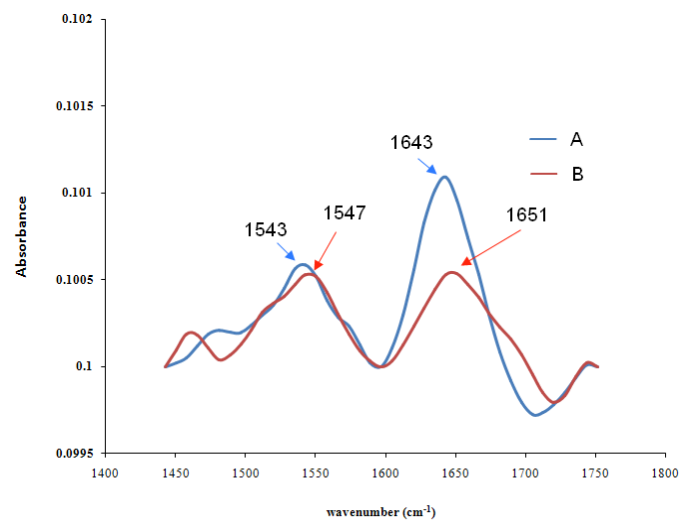

Figure 11: FT-IR spectra in the region of $1400-1800 \mathrm{~cm}^{-1}$ for $1.0 \times 10^{-6} \mathrm{M}$ of (A) free HAS (blue line) and, (B) LOP-HSA complex 1:1 ratio between ligand and $\mathrm{HSA}$ (red line) at $\mathrm{pH} 7.4$.

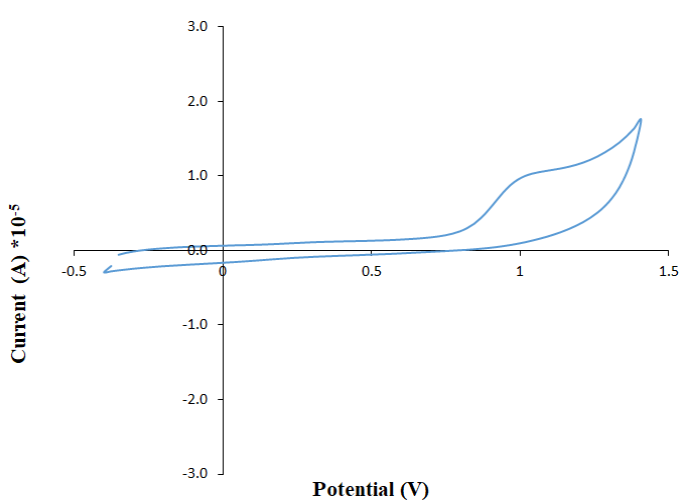

Figure 12: Cyclic voltammogram of $1 \mu \mathrm{M} \mathrm{LOP.}$

LOP to serum albumin on the amide II region [36,37]. The changes in peak positions and peak shapes demonstrate that LOP induces a slight change in the secondary structure of HSA.

\section{Voltammetric studies}

Figure 12 report the cyclic voltammogram of LOP on a GCE with a peak potential of $+1 \mathrm{~V}$. During the all electrochemical experiments, the GCE was activated in the same scan potential range. The experimental results indicated well-defined and reproducible anodic peak.

The voltammograms exhibit one well-defined anodic peak at about $1000 \mathrm{mV}$, with no peak on the reverse scan, suggesting the irreversible nature of the electrode reaction. The presence of azometine reducible group $(-\mathrm{C}=\mathrm{N}-)$ in the structure of LOP is probably responsible for the observed reduction wave.

The cyclic voltammograms of HSA (with concentration of $1 \times 10^{-5}$ ) at the working electrode in the presence of different amount of LOP are also shown in Figure 13A. As it can be seen, when LOP was added to the HSA solution, the anodic and cathodic peak currents are decreased (Figure 13A curve I), implying that an electrochemical non-active complex could have been formed [38]. The formation of the complex resulted in the decrease of the equilibrium concentration of HSA in solution, leading to the decrease of the peak current. The obtained result is in consistent with the above results from fluorescence, UV-Vis and FT-IR measurements.

Figure $13 \mathrm{~B}$ indicates that the electron transfer between the 
$\mathrm{K}_{3} \mathrm{Fe}(\mathrm{CN})_{6} / \mathrm{K}_{4} \mathrm{Fe}(\mathrm{CN})$ Redox probe and the electrode was slightly blocked after binding LOP-HSA complex onto the surface of electrode. Such an electron-transfer block to the redox probe was amplified by precipitation generated on the working electrode through formation reaction between LOP and HSA.

The complex of LOP-HSA has large size like other drug-HSA complexes, thus typically it takes time for different small molecules like drugs to reach equilibrium with protein. For the complex of LOP-HSA, further decrease in peak currents was observed at longer times that reached a constant value after about $120 \mathrm{~s}$. Consequently, each mixture of LOP and HSA was allowed to stand for $120 \mathrm{~s}$ to approach the equilibrium conditions. If we look at LOP structure greater scrutiny, the electroactive parts of the LOP i.e., the $\left(\mathrm{CH}_{3}\right) 2 \mathrm{~N}$ and $\mathrm{Cl}$ groups, were embedded within the protein binding pocket. This binding led to preclude its interaction at the electrode surface and as a result its participation in the Redox reaction. In addition, the concentration of free LOPS at the electrode surface diminished during the electrochemical experiments, and therefore, the peak current (both anodic and cathodic) was also decreased. As a result, the observed slight potential shifts of the oxidation and reduction peaks can be attributed to the alterations of the molecular environment of LOP as a result of its interaction with protein.

As explained in literature, EIS is a flexible and efficient technique to observing dynamic ligand-protein interactions [39].

In EIS experiment, the certain quantity of LOP was added to the electrochemical cell with the fixed concentration of serum albumin $\left(1.0 \times 10^{-5} \mathrm{M}\right)$, so the change of Rct value was indicative of the change of surface binding of LOP and blocking. As it can be seen in Figure 13B, Rct increases in a dose-dependent after the addition of LOP. This phenomenon indicates LOP binds to HSA and form a non-active electrochemical complex on the surface of electrode accompanying lead to increase in the resistance of the electrode.

The value of the electrode was estimated by its diameter. Figure 13B shows that electron-transfer resistance (Rct) increased in a dosedependent manner after addition of LOP, indicating the formation of a non-active electrochemical complex, consistent with aforementioned cyclic voltammetry results.

\section{Dynamic light scattering analysis}

DLS is a helpful method for the measurement of important information of biological macromolecules in solution. In the DLS method, the hydrodynamic diameter and polydispersity of biological macromolecules is determined. It could be reported that DLS is one
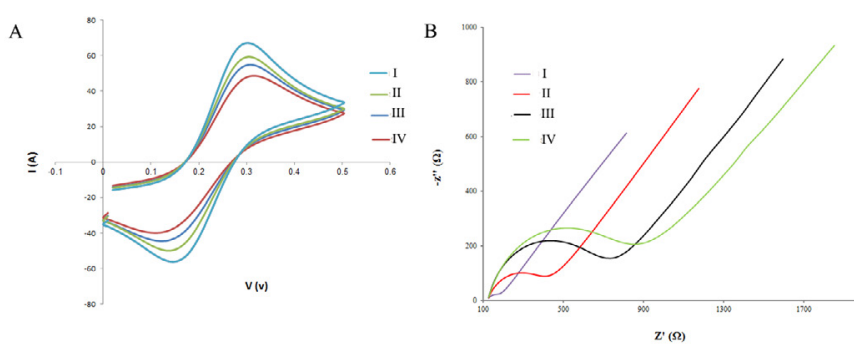

Figure 13: $(A)$ Cyclic voltammograms of $\operatorname{HSA}\left(1 \times 10^{-5} \mathrm{M}\right)$ at working electrode with tacc $=90 \mathrm{~s}$ and the potential scan rate is $100 \mathrm{mV} \mathrm{s}^{-1}$ in the presence of LOP with concentration of (I) 0 , (II) $5 \times 10^{-6} \mathrm{M}$, (III) $1 \times 10^{-5} \mathrm{M}$ (IV) $5 \times 10^{-5} \mathrm{M}$ (B) relative $R c t(i) / R c t$ intensity for generated electrode as a function of the fixed concentration of serum albumin $\left(1 \times 10^{-5} \mathrm{M}\right)$ and different concentration of LOP (I) $0 \mathrm{M}$, (II) $5 \times 10^{-6} \mathrm{M}$, (III) $1 \times 10^{-5} \mathrm{M}$ (IV) $5 \times 10^{-5} \mathrm{M}$.

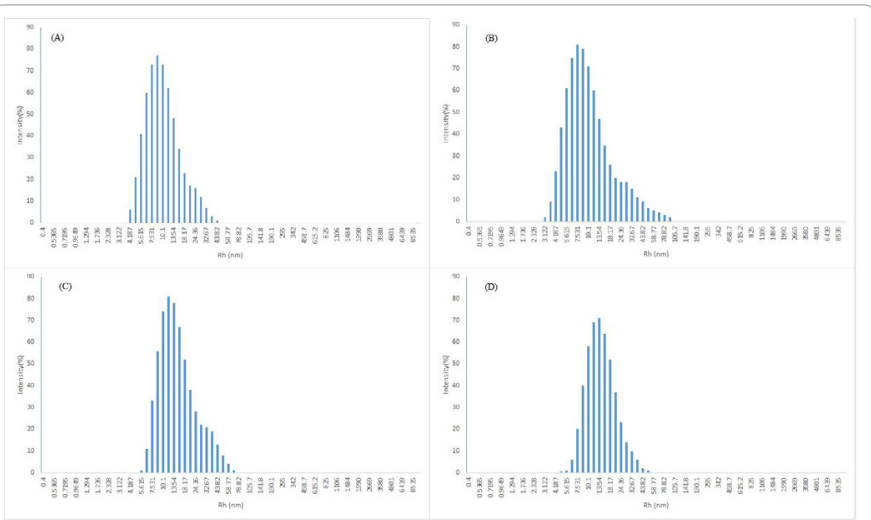

Figure 14: Hydrodynamic radii pattern of HAS $\left(2 \times 10^{-5} \mathrm{M}\right)(\mathrm{A})$ in the absence of LOP (B) $[\mathrm{LOP}] /[\mathrm{HAS}]$ and presence of HSA+CBDA.

of the most useful method for determining and monitoring the size of macromolecules and complex, and for further investigation of interactions between small molecules and biomacromolecules [40,41].

In the present work, DLS was used to measure the hydrodynamic radii (Rh) of free HSA and HSA-LOP complex. In our work, the observed $\mathrm{Rh}$ of native HSA and complex are plotted in Figure 14. The Rh value of native HSA was $8.85 \mathrm{~nm}$. However, increasing the concentration of LOP by the molar ratio of HSA:LOP=1:1, 1:5 and 1:10 (Figure 14B-14D) leads to increase in the Rh values of HSA-LOP complex. The increasing in $\mathrm{Rh}$ value may be attributed to the complexion of LOP with HSA, which results in shrinkage of molecular volume due to conformational changes. Probably, the interaction of LOP with HSA, in such a way that the solvent shell surrounding the HSA, caused rearrangement and increasing in hydrodynamic radii. Furthermore, the conformational alterations of the complex which is probably accrued by LOP binding caused increasing of hydrodynamic radii.

\section{Molecular docking}

Molecular docking was performed in order to find the available interaction sites, the preferred position and orientation of LOP as a ligand on HSA.

As discussed in literature [10,11], crystallographic data indicate that serum albumin, is a globular protein of 585 amino acids, comprises three homologous $\alpha$-helical domains that are structurally similar: (denoted I, II, and III): domain I includes residues 1 to 195 , domain II includes 196 to 383, and domain III includes 384 to 585; each domain has two subdomains ( $\mathrm{A}$ and $\mathrm{B}$ ) that assemble to form heart shaped molecule. The main sites of ligand binding of HSA are located in hydrophobic cavities in subdomains IIA and IIIA, which are corresponding to sites I and II, respectively. It must be noted that a tryptophan residue (Trp-214) is located in subdomain IIA of serum albumin [12]. The best-docked conformation of the LOP (Figure 14) was studied and is shown in the Figure 14. It was found that LOP was located within subdomain IIA binding site with mainly hydrophobic properties, and it is in close proximity to hydrophobic residues, such as Phe 206, Ala 210, Lys212, Ala 213, Trp214, Leu327, Ala 350, Leu347, Leu 481 and VAL 482 of subdomain IIA of HSA [40]. The result of computational docking strongly confirmed the importance of hydrophobic interaction between LOP and subdomain IIA binding pocket. Also, the interaction investigation revealed that there is a potential for hydrogen bonding by LOP with Val 482 in the binding site of serum albumin, $(3.21 \AA)$. Similarly, one hydrogen bonding can be form between the Asp 324 with a distance of $3.24 \AA$. The docking 
results shown in Figure 14 also implied that LOP binds to serum albumin binding site I through several interactions including: $\pi-\pi$ stacking interactions between phenyl moiety of LOP and the aromatic moiety of Phe 206, hydroxyl group as an partially acidic group and basic side chain residue of Asp 324, and partially hydrogen bonding interactions between some of residues (Lys 351, Arg 348, Arg 209, Asp 483, and Ser 400) and $\mathrm{N}$ and $\mathrm{O}$ atoms of LOP.

Therefore, in brief, the results of docking show that the interaction between LOP and serum albumin are dominated by hydrophobic forces, which is agreement with the experimental data. It was important for mentioned that the Trp 214 residue of serum albumin is in close proximity to the ligand molecule (Figure 15). This finding provides a good structural basis to explain the efficient fluorescence quenching of HSA emission in the presence of LOP. Our results have shown that LOP can interact with HSA at site I in subdomain IIA with mainly hydrophobic interactions, which is consistent with the previously published data on other ligand molecules.

\section{Molecular dynamics simulation}

Experimental approaches cannot provide complete and comprehensive understanding of atomistic details of ligand binding process that happen in some cases in mere millionths of second. Molecular dynamics (MD) simulation is one of the best methods for understanding the physical basis of ligand binding process, changes in structure and function of macromolecules in atomistic details. MD calculation can be also used as a powerful tool for study the binding structures and recognize the best binding mode [12,41-44]. To study the stability of the studied system (HSA, LOP, water, ions, etc.), different properties like Root mean square deviation (RMSD), Root mean square fluctuation (RMSF) and Radius of gyration (Rg) were estimated to find the stability, rigidity, and micro-environmental changes for HSA and LOP-HSA complex system. As well, the stability of LOP-HSA complex confirmed the credibility of the docking result (Figure 16), where LOP bound to serum albumin at binding pocket I, which is the IIA subdomain, were used for MD simulations.

The final docked complex of HSA and LOP was used for MD Simulation. The potential energy plots obtained from MD simulation represented that all simulated interactions were stabilized throughout the MD simulation (data not shown).

Therefore, the two systems, free HSA and LOP-HSA complex, were submitted to $50 \mathrm{~ns}$ long MD simulations in explicit solvent.

After energy minimization and slow heating to reach equilibrium at room temperature ( $300 \mathrm{~K}$ and $1 \mathrm{bar}$ ), the RMSD values of atoms in free

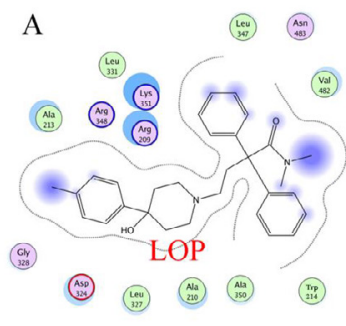

(iiv)

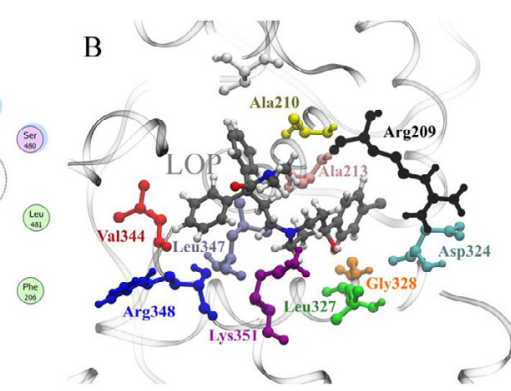

Figure 15: Molecular docked model of LOP located within the binding pocket in subdomain IIA of HSA (A) 2D representation of involved residues and important interactions (B) $3 D$ representation of involved residues and LOP in Licorice representation.
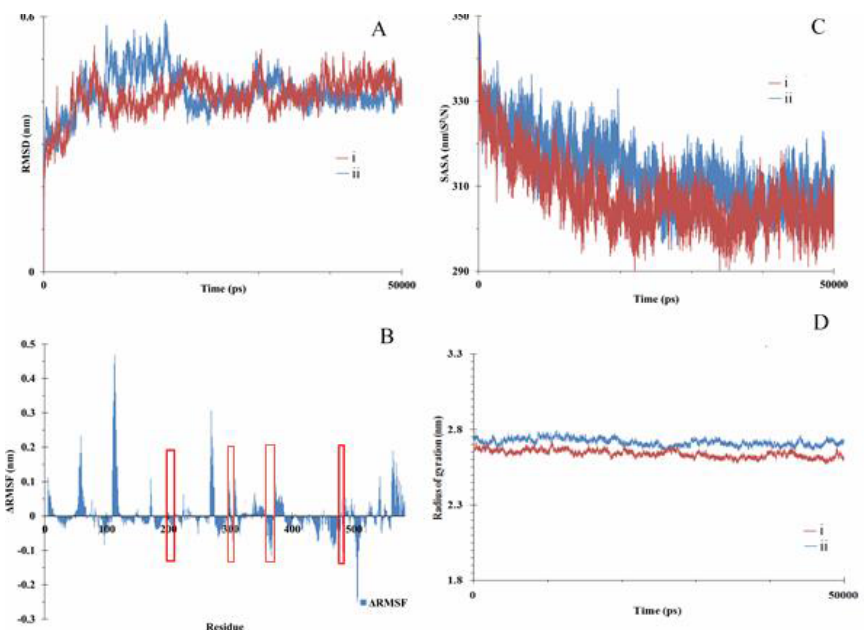

Figure 16: (A) Root mean square deviations (RMSD) of $C \alpha$ atoms as a function of time. (i) free HSA and (ii) LOP-HSA complex (B) Change in RMSF fluctuations of $C \alpha$ atoms extracted from MD simulation between free HSA and LOP-HSA complex, residues involved in the drug contacting are highlighted by red rectangles. (C) Graphical representation of the sequence of solvent accessible surface area (SASA) changes during MD simulation. For (i) free HSA and (ii) LOP-HSA complex (D) Radius of gyration of (i) free HSA and (ii) LOPHSA complex for 50 ns MD simulation for (i) free HSA and (ii) LOP-HSA complex.

HSA and LOP-HSA complex were plotted from 0 to 50000 ps as shown in Figure 16A. Analysis of the Figure 16A indicates that the RMSD of both systems reaches equilibration and oscillates around in average value after $20000 \mathrm{ps}$ simulation time. The RMSD values of atoms in free HSA and LOP-HSA complex were calculated from a 20000-50000 ps trajectory, where the data points were fluctuated for free HSA, 0.428 ( $\pm 0.028 \mathrm{~nm})$ and LOP-HSA complex $0.41(0.023 \mathrm{~nm})$, respectively. It is often considered that small RMSD values of a simulation indicate a stable state of the system. This highlights the stable binding of the LOP with HSA.

To further characterize the dynamic behaviour or determine structural stability of serum albumin and how it is affected by formation of complex of LOP-HSA, the change in RMSF values of the Ca atoms in HSA induced by the LOP binding was monitored using MD trajectories. As well, during the investigation of RMSF, the stability of system proved the credibility of the docking result. Graph $\left(\triangle \mathrm{RMSF}=\mathrm{RMSF}_{L O P-H S A}\right.$ $\mathrm{RMSF}_{\mathrm{HSA}}$ ) was plotted (Figure 16B) to obtain the residue mobility and it was found that the LOP-HSA complex structure exhibited different fluctuation than the free HAS in some regions.

It can be seen from Figure 16B that the residues involved in the drug contacting are less flexible (with negative $\triangle \mathrm{RMSF}$ ) as LOP is bound (regions highlighted by red rectangles).

Interestingly, the other regions which are not subjected to the contacts from the drug become more flexible in the drug-bound state. These two phenomena compensate with each other and consequently the average flexibility of the protease might be not highly influenced by the drug binding (average RMSF for free HSA is 0.194 and for LOPHSA is $0.198 \mathrm{~nm}$ ).

Minor differences were observed in the fluctuations of these systems, which could be ascribed to minor secondary structure variations. Ligand binding often occurs with losses in conformational entropy [45]. Ligand binding requires the formation of well-defined, non-bonded interactions, which is often associated with losses in conformational entropy [46]. 


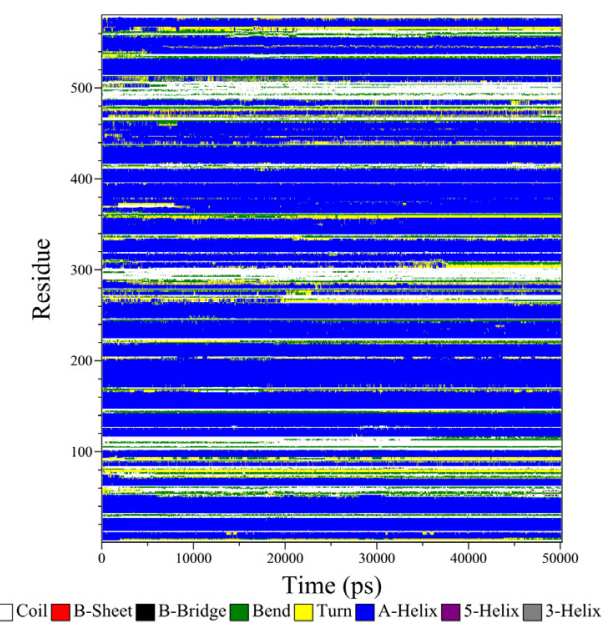

Figure 17: Variation of the secondary structure of HSA versus time for the LOP-HAS complex.

All of the residues in the HSA binding site (e.g. residues 206213, residues 324-331, 347-354 and residues 480-482) (The region is highlighted with red bar) that binds with LOP show relative small degree of flexibility when compared with free HSA, indicating that residues locating in binding site seem to be more rigid as a result of binding to LOP.

Solvent accessible surface area (SASA) can be considered as an important feature which defines the surface area of a macromolecule such as protein that is accessible to a solvent probe. In this method the surface area is monitored by using a $1.4 \AA$ radius for the probe.

The change of SASA of the free HSA and LOP-HSA with time is shown in Figure 16C.

As it can be seen, LOP-HSA complex showed relatively higher values of SASA with time, while free HSA structure showed smaller values. The average value of SASA during the simulation time period from a 20000-50000 ps trajectory in free HSA and LOP-HSA complex is calculated (309 $\mathrm{nm}^{2}$ and $303 \mathrm{~nm}^{2}$, respectively). For two studied system, the SASA is decreased from that for starting structure (from about $318 \mathrm{~nm}^{2}$ to $298 \mathrm{~nm}^{2}$ for free HSA and from about $317 \mathrm{~nm}^{2}$ to 308 $\mathrm{nm}^{2}$ for LOP-HSA complex) along the trajectory showed the effective binding of LOP to HSA.

Also, in the present study, the radius of gyration $(\mathrm{Rg})$ values was determined for free HSA and LOP-HSA complex as shown in Figure 16D. It must be noted that $\mathrm{Rg}$ is the property of overall dimension of macromolecule during simulation and considered as estimation of mass weighted root mean square distance from the center of mass of all atoms. In other words, $\mathrm{Rg}$ is used as a parameter that implies the structure backbone stability, rigidity, and compactness. From the results, it could be mentioned that Rg was slightly greater than for HSA-LOP complex compared to free HSA. In case of free HSA complex, it was observed that the Rg value started with $1.66 \mathrm{~nm}$ and is fixed till the end of production time. The other system, HSA-LOP complex structure showed $\mathrm{Rg}$ range of 2.66 to $2.67 \mathrm{~nm}$. Rg value was slightly deviated in the LOP-HSA complex and confirmed the partially change the structure of HSA.

The secondary structure of serum albumin was investigated also during MD simulation too. Figure 17 showed that when serum albumin interacted with LOP, its secondary structure was not significantly changed. Therefore, completely deactivation of serum albumin in LOPHSA conjugate seemed not due to the secondary structure change. Meanwhile, the binding site of LOP to serum albumin were deeply buried into the hydrophobic core.

\section{Conclusion}

In this paper, we studied the interaction of the LOP with human serum albumin by combination of different experimental and computational methods such as fluorescence spectroscopy, UV-Vis, FT-IR, voltammetry, Impedance Spectroscopy, molecular docking and molecular dynamics simulation. The results suggested that the quenching of fluorescence of HSA resulted mainly by static mechanism as there was no significant change in lifetime of HSA. The positive value of $\Delta \mathrm{H}$ and the positive value of $\Delta \mathrm{S}$ show that hydrophobic interactions play major roles in the binding of the LOP and protein. The UV-Vis and FT-IR spectra of HSA in the presence of LOP showed that the binding of drug causes the slight conformational change and secondary structure alteration in protein. Also docking results suggested that LOP can bind in the large hydrophobic cavity of subdomain IIA, mainly by the hydrophobic interaction to hydrophobic residues, such as Lys212, Trp214, Val235, Val325, VAL231, VAL216, Phe228, and Gly228.

From the present study it is found that LOP interacts with HSA and the binding affinity of LOP-HSA is fairly high, consequently this may result in excessive accumulation of LOP in blood.

\section{Acknowledgement}

Authors appreciate from the research council of Kermanshah University of Medical Sciences for financial supports (approved proposal No. 95308). This work was performed in partial fulfilment of the requirement for Pharm. D of Mrs. Fatemeh Rahmati.

\section{References}

1. Pharmacopoeia B (2007) The stationery office on behalf of the medicines and healthcare products regulatory agency (MHRA). London. United Kingdom.

2. DeHaven-Hudkins D, Burgos LC, Cassel J, Daubert J, DeHaven R, et al. (1999) Loperamide (ADL 2-1294), an opioid antihyperalgesic agent with peripheral selectivity. J Pharmacol Exp Ther 289: 494-502.

3. Nozaki-Taguchi N, Yaksh TL (1999) Characterization of the antihyperalgesic action of a novel peripheral mu-opioid receptor agonist-loperamide. The Journal of the American Society of Anesthesiologists 90: 225-234.

4. Regnard C, Twycross R, Mihalyo M, Wilcock A (2011) Loperamide. Journal of pain and symptom management 42: 319-323.

5. Dreverman J, POEL A (1995) Loperamide oxide in acute diarrhoea: a doubleblind, placebo-controlled trial. Alimentary Pharmacology \& Therapeutics 9 441-446.

6. Peters Jr T (1995) All about albumin: biochemistry, genetics, and medical applications. Academic press.

7. Peters T (1985) Serum albumin. Advances in Protein Chemistry 37: 161-245.

8. Kragh-Hansen U (1985) Relations between high-affinity binding sites of markers for binding regions on human serum albumin. Bio Chem J 225: 629-638.

9. Curry S, Mandelkow H, Brick P, Franks N (1998) Crystal structure of human serum albumin complexed with fatty acid reveals an asymmetric distribution of binding sites. Nat Struct Mol Biol 5: 827-835

10. Shahlaei M, Rahimi B, Ashrafi-Kooshk MR, Sadrjavadi K, Khodarahmi R (2015) Probing of possible olanzapine binding site on human serum albumin Combination of spectroscopic methods and molecular dynamics simulation. Journal of Luminescence 158: 91-98.

11. Moradi N, Ashrafi-Kooshk MR, Ghobadi S, Shahlaei M, Khodarahmi R (2015) Spectroscopic study of drug-binding characteristics of unmodified and pNPA-based acetylated human serum albumin: Does esterase activity affect microenvironment of drug binding sites on the protein? Journal of Luminescence 160: 351-361. 
Citation: Sadrjavadi K, Rahmati F, Jafari F, Moradi S, Nowroozi A, et al. (2017) A Study on the Binding of Loperamide to Human Serum Albumin Using Combination of Computational and Experimental Methods. Biochem Anal Biochem 6: 346. doi: 10.4172/2161-1009.1000346

12. Shahlaei M, Rahimi B, Nowroozi A, Ashrafi-Kooshk MR, Sadrjavadi K et al. (2015) Exploring binding properties of sertraline with human serum albumin Combination of spectroscopic and molecular modeling studies. Chem-Bio Interact 242: 235-246.

13. Trott O, Olson AJ (2010) AutoDock Vina: improving the speed and accuracy of docking with a new scoring function, efficient optimization, and multithreading. Journal of Computational Chemistry 31: 455-461.

14. Morris GM, Huey R, Lindstrom W, Sanner MF, Belew RK, et al. (2009) AutoDock4 and AutoDockTools4: Automated docking with selective receptor flexibility. Journal of Computational Chemistry 30: 2785-2791

15. Gasteiger J, Marsili M (1980) Iterative partial equalization of orbital electronegativity-a rapid access to atomic charges. Tetrahedron 36: 32193228.

16. Singh UC, Kollman PA (1984) An approach to computing electrostatic charges for molecules. Journal of Computational Chemistry 5: 129-145.

17. Morris GM, Goodsell DS, Halliday RS, Huey R, Hart WE, et al. (1998) Automated docking using a Lamarckian genetic algorithm and an empirical binding free energy function. Journal of Computational Chemistry 19: 1639-1662.

18. Humphrey W, Dalke A, Schulten K (1996) VMD: visual molecular dynamics. J Mol Graphics 14: 33-38.

19. Abraham MJ, Murtola T, Schulz R, Páll S, Smith JC, et al. (2015) GROMACS: High performance molecular simulations through multi-level parallelism from laptops to supercomputers. SoftwareX 1: 19-25.

20. Berendsen HJ, Postma JP, van Gunsteren WF, Hermans J (1981) Interaction models for water in relation to protein hydration. In: Intermolecular forces. Springer, pp: 331-342.

21. Canzar S, El-Kebir M, Pool R, Elbassioni K, Malde AK, et al. (2013) Charge group partitioning in biomolecular simulation. J Comput Biol 20: 188-198.

22. Quiming NS, Vergel RB, Nicolas MG, Villanueva JA (2005) Interaction of bovine serum albumin and metallothionein. J Health Sci 51: 8-15.

23. Hu Y-J, Liu Y, Shen X-S, Fang X-Y, Qu S-S (2005) Studies on the interaction between 1-hexylcarbamoyl-5-fluorouracil and bovine serum albumin. Journal of molecular structure 738: 143-147

24. Sułkowska $A(2002)$ Interaction of drugs with bovine and human serum albumin Journal of molecular structure $614: 227-232$

25. Li Z, Shang LP, Deng H (2009) Extraction of characteristic parameters of threedimensional fluorescence spectra of tyrosine and tryptophan. Spectroscopy and Spectral Analysis 29: 1925-1928.

26. Dewey TG (1991) Biophysical and biochemical aspects of fluorescence spectroscopy. Springer

27. Abou-Zied OK, Al-Shihi OI (2008) Characterization of subdomain IIA binding site of human serum albumin in its native, unfolded, and refolded states using small molecular probes. J Am Chem Soc 130: 10793-10801.

28. Lehrer S (1971) Solute perturbation of protein fluorescence. Quenching of the tryptophyl fluorescence of model compounds and of lysozyme by iodide ion. Biochemistry 10: 3254-3263.

29. Zhang Y-Z, Zhou B, Liu Y-X, Zhou C-X, Ding X-L, et al. (2008) Fluorescence study on the interaction of bovine serum albumin with p-aminoazobenzene. Journal of Fluorescence 18: 109-118.
30. Ross PD, Subramanian S (1981) Thermodynamics of protein association reactions: forces contributing to stability. Biochemistry 20: 3096-3102.

31. Poulli KI, Chantzos NV, Mousdis GA, Georgiou CA (2009) Synchronous fluorescence spectroscopy: tool for monitoring thermally stressed edible oils. J Agric Food Chem 57: 8194-8201.

32. Varlan A, Hillebrand M (2010) Bovine and human serum albumin interactions with 3-carboxyphenoxathiin studied by fluorescence and circular dichroism spectroscopy. Molecules 15: 3905-3919.

33. Miller J (1979) Recent advances in molecular luminescence analysis. In: Proc Anal Div Chem Soc Pp : 203-208.

34. Lakowicz JR (2013) Principles of fluorescence spectroscopy. Springer Science \& Business Media

35. Byler DM, Susi $H$ (1986) Examination of the secondary structure of proteins by deconvolved FTIR spectra. Biopolymers 25: 469-487.

36. Susi H, Byler DM (1986) [13] Resolution-enhanced fourier transform infrared spectroscopy of enzymes. Methods Enzymol 130: 290-311

37. Krimm S, Bandekar J (1986) Vibrational spectroscopy and conformation of peptides, polypeptides, and proteins. Advances in Protein Chemistry 38: 181364

38. Xu Z-Q, Zhou B, Jiang F-L, Dai J, Liu Y (2013) Interaction between a cationic porphyrin and ctDNA investigated by SPR, CV and UV-vis spectroscopy. Colloids Surf B Biointerfaces 110: 321-326.

39. Tu B, Wang Y, Mi R, Ouyang Y, Hu Y-J (2015) Evaluation of the interaction between naringenin and human serum albumin: Insights from fluorescence spectroscopy, electrochemical measurement and molecular docking Spectrochimica Acta Part A: Molecular and Biomolecular Spectroscopy 149: 536-543.

40. Carter DC, He X-M, Twigg PD, Casale E (1991) Three-dimensional structure of human serum albumin. JSTOR.

41. Shahlaei M, Rahimi B, Nowroozi A, Ashrafi-Kooshk MR, Sadrjavadi K, et al. (2016) Corrigendum to "Exploring binding properties of sertraline with human serum albumin: Combination of spectroscopic and molecular modeling studies" [Chem. Biol. Interact. 242 (2015) 235-246]. Chemico-biological interactions 252: 150.

42. Shahlaei M, Madadkar-Sobhani A, Mahnam K, Fassihi A, Saghaie L, et al. (2011) Homology modeling of human CCR5 and analysis of its binding properties through molecular docking and molecular dynamics simulation. Biochimica et Biophysica Acta (BBA)-Biomembranes 1808: 802-817.

43. Shahlaei M, Madadkar-Sobhani A, Fassihi A, Saghaie L (2011) Exploring a model of a chemokine receptor/ligand complex in an explicit membrane environment by molecular dynamics simulation: the human CCR1 receptor. Journal of chemical information and modeling 51: 2717-2730.

44. Shahlaei M, Fassihi A, Papaleo E, Pourfarzam M (2013) Molecular dynamics simulation of chemokine receptors in lipid bilayer: a case study on C-C chemokine receptor type 2. Chemical biology \& drug design 82: 534-545.

45. Cooper A (1999) Thermodynamic analysis of biomolecular interactions. Current Opinion in Chemical Biology 3: 557-563.

46. Kariv I, Cao H, Oldenburg KR (2001) Development of a high throughput equilibrium dialysis method. Journal of pharmaceutical sciences 90: 580-587. 